\title{
Long-term survival in patients with long-segment complex meningiomas occluding the dural venous sinuses: illustrative cases
}

\author{
Zhishuo Wei, MS, Arka N. Mallela, MD, MS, Andrew Faramand, MD, Ajay Niranjan, MD, MBA, and L. Dade Lunsford, MD \\ Department of Neurological Surgery, University of Pittsburgh Medical Center, Pittsburgh, Pennsylvania
}

BACKGROUND Invasive sagittal sinus meningiomas are difficult tumors to cure by resection alone. Stereotactic radiosurgery (SRS) can be used as an adjuvant management strategy to improve tumor control after incomplete resection.

OBSERVATIONS The authors reported the long-term retrospective follow-up of two patients whose recurrent parasagittal meningiomas eventually occluded their superior sagittal sinus. Both patients underwent staged radiosurgery and fractionated radiation therapy to achieve tumor control that extended to 20 years after their initial surgery. After initial subtotal resection of meningiomas that had invaded major cerebral venous sinuses, adjuvant radiosurgery was performed to enhance local tumor control. Over time, adjacent tumor progression required repeat SRS and fractionated radiation therapy to boost long-term tumor response. Staged multimodality intervention led to extended survival in these patients with otherwise unresectable meningiomas.

LESSONS Multimodality management with radiosurgery and fractionated radiation therapy was associated with long-term survival of two patients with otherwise surgically incurable and invasive meningiomas of the dural venous sinuses.

https://thejns.org/doi/abs/10.3171/CASE21116

KEYWORDS stereotactic radiosurgery; dural venous sinuses; radiation therapy

Meningiomas are the most common primary benign intracranial neoplasm, with an annual incidence of 15 cases per 100,000 persons. ${ }^{1}$ Complete resection of the tumor and its dural origin remains the primary treatment option for larger, growing, or symptomatic meningiomas. Prolonged survival of patients with meningiomas that occlude venous sinuses is rare. ${ }^{2-4}$ Even histologically slow-growing grade I meningiomas can exhibit more aggressive behavior when they invade dura, bone, or critical venous sinuses. ${ }^{5}$ Although resection is an important initial strategy in patients with symptomatic parasagittal meningioma, surgery alone results in 5-, 10-, and 15-year progression-free survival rates of $50 \%, 40 \%$, and $30 \%$, respectively. ${ }^{6}$ Adjuvant stereotactic radiosurgery (SRS) for residual tumors improves long-term tumor control and neurological outcomes in such patients. ${ }^{7}$

We present the long-term outcomes of two patients whose initially low-grade parasagittal meningiomas invaded or occluded venous sinus outflows. Multimodality management using a combination of surgery, SRS, and radiation therapy resulted in prolonged survival in these patients.

\section{Illustrative Cases}

\section{Case 1}

A 55-year-old woman presented with headache, imbalance, and a right inferior visual field defect. Magnetic resonance imaging (MRI) demonstrated a large parasagittal meningioma that was invading the sagittal and transverse sinuses bilaterally, resulting in extensive left hemispheric edema. Angiography confirmed that the tumor filled the superior sagittal sinus (SSS) and the torcula. The patient underwent initial tumor embolization followed by craniotomy and partial tumor resection of the parasagittal mass. Pathology revealed a World Health Organization (WHO) grade I meningothelial meningioma with low proliferation index. Postoperative imaging demonstrated residual tumor in the sagittal sinus, torcula, and both transverse sinuses (Fig. 1).

ABBREVIATIONS GK = Gamma Knife; IMRT = intensity-modulated radiation therapy; MRI = magnetic resonance imaging; SRS = stereotactic radiosurgery; SSS = superior sagittal sinus; $W H O=$ World Health Organization.

INCLUDE WHEN CITING Published May 17, 2021; DOI: 10.3171/CASE21116.

SUBMITTED February 23, 2021. ACCEPTED March 3, 2021.

(C) 2021 The authors, CC BY-NC-ND 4.0 (http://creativecommons.org/licenses/by-nc-nd/4.0/). 


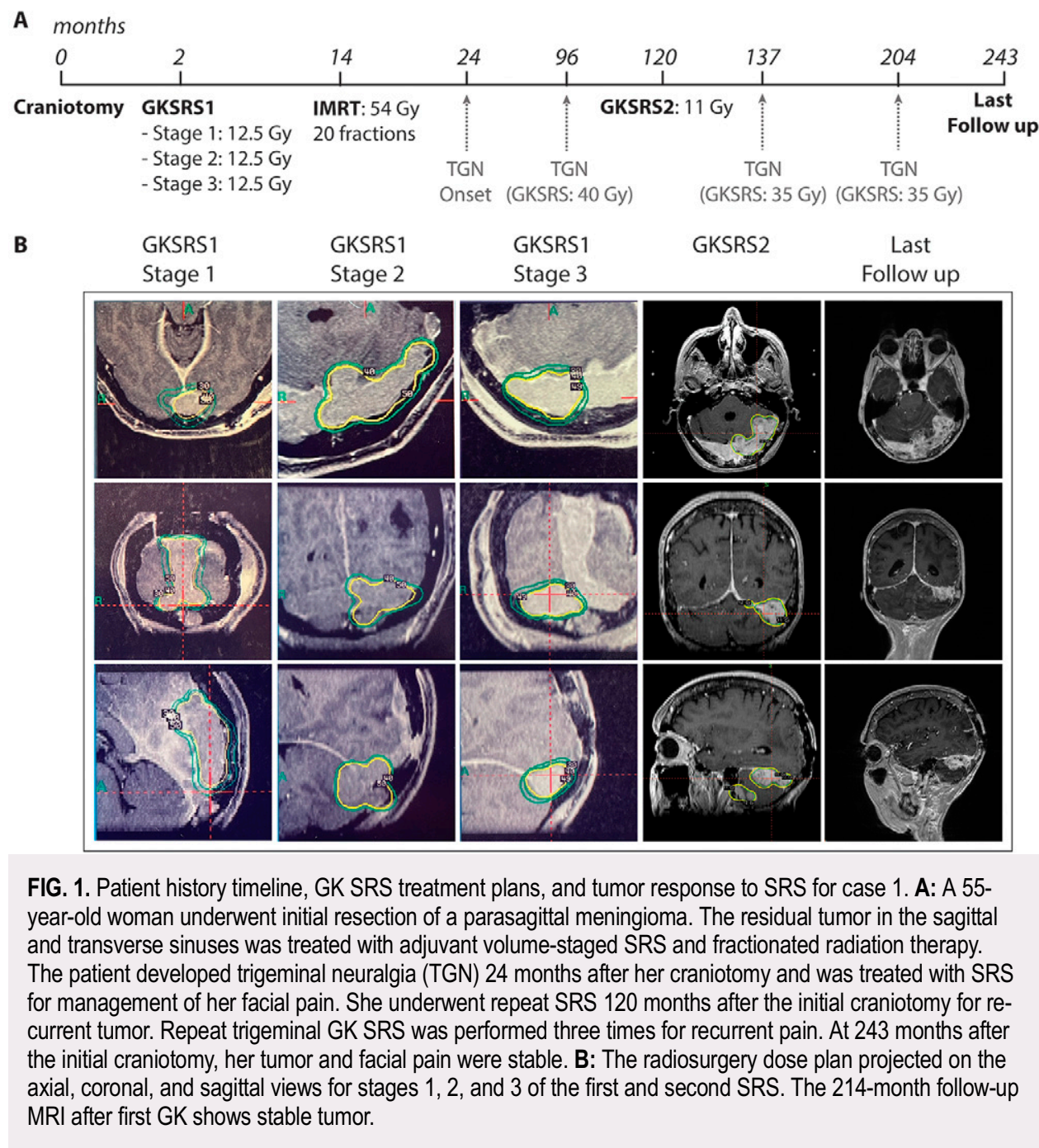

Images obtained 2 months after resection demonstrated progression of the tumor. Because of the large volume of residual tumor, SRS was performed in three stages over a 3-month period. A margin dose of 12.5 Gy was delivered to the $50 \%$ isodose line during each volume-staged SRS.

Because of suspected tumor progression 1 year after the last radiosurgery procedure, the patient underwent fractionated intensity-modulated radiation therapy (IMRT) (54 Gy, 20 fractions). She developed classic right-sided trigeminal neuralgia 2 years later. We suspected that a dilated petrosal vein led to trigeminal nerve venous compression. Her pain was managed initially with medication. Because of severe pain exacerbation 9 years after onset, she underwent SRS directed at the root entry zone of the trigeminal nerve (4-mm collimator, 80-Gy maximum dose). Her facial pain gradually improved, but two additional trigeminal Gamma Knife (GK) SRS procedures were performed because of recurrent pain. Additional tumor progression associated with cerebellar edema was treated with additional SRS (11 Gy, 50\% isodose), and bevacizumab was begun. Twenty-one years after her initial craniotomy, the patient remains stable neurologically while continuing maintenance bevacizumab therapy.

\section{Case 2}

A 49-year-old woman presented with speech disorder, headaches, and nausea. MRI demonstrated an $80-\mathrm{cm}^{3}$ left frontal parasagittal meningioma that required initial craniotomy and tumor removal. The pathology was a meningothelial WHO grade I meningioma. Two years after resection, the patient reported headaches and short-term memory loss. Tumor progression in the anterior sagittal sinus was managed with SRS (Fig. 2). Eighteen months after that procedure, tumor progression in the falx was treated using repeat radiosurgery. Three years after the second treatment, tumor progression in the mid and posterior SSS was treated with a third radiosurgical procedure. Because of additional tumor progression, 6 years later the patient underwent fractionated IMRT (54 Gy, 30 fractions) as well as a fourth SRS. Eighteen years after her craniotomy, the patient remained neurologically stable with no neurological signs or symptoms. 
A months

\begin{tabular}{|c|c|c|c|c|c|c|}
\hline $\begin{array}{l}0 \\
L\end{array}$ & $\begin{array}{c}25 \\
1 \\
\end{array}$ & $\begin{array}{c}36 \\
1\end{array}$ & $\begin{array}{c}59 \\
1 \\
\end{array}$ & $\begin{array}{c}92 \\
1\end{array}$ & $\begin{array}{c}93 \\
1\end{array}$ & 216 \\
\hline Craniotomy & $\begin{array}{l}\text { GKSRS1 } \\
13 \mathrm{~Gy}\end{array}$ & $\begin{array}{l}\text { GRSRS2 } \\
15 \mathrm{~Gy}\end{array}$ & $\begin{array}{l}\text { GRSRS3 } \\
12 \mathrm{~Gy}\end{array}$ & $\begin{array}{l}\text { IMRT: } 54 \text { Gy } \\
30 \text { fractions }\end{array}$ & $\begin{array}{l}\text { GKSRS4 } \\
14 \text { Gy }\end{array}$ & $\begin{array}{l}\text { Last } \\
\text { Follow up }\end{array}$ \\
\hline
\end{tabular}

B GKSRS 1 GKSRS 2 GKSRS 3 GKSRS 4 Last Follow up
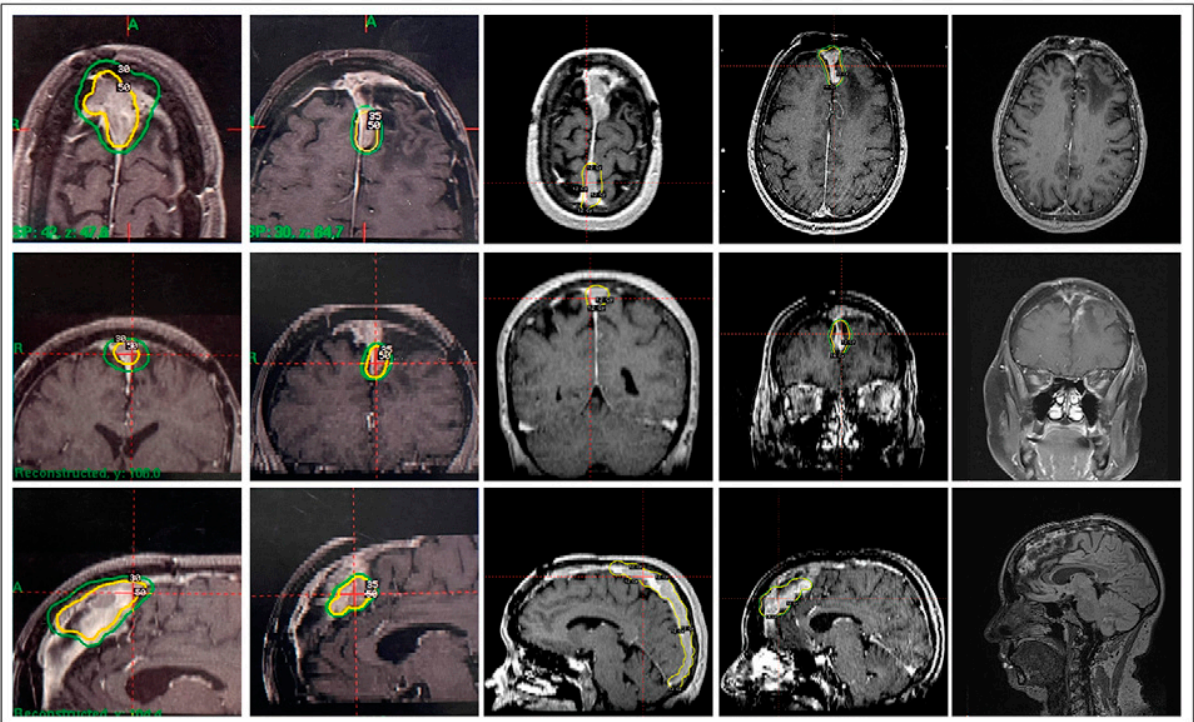

FIG. 2. Patient history timeline, GK SRS treatment plans, and tumor response to SRS for case 2. A: A 49year-old woman underwent initial resection of a left frontal meningioma. The residual and recurrent tumors in the SSS were treated with four SRS procedures and boosted with radiation therapy over an interval of 216 months. The patient was neurologically and functionally stable 216 months after her initial craniotomy. B: The radiosurgery dose plans projected on the axial, coronal, and sagittal views of the four SRS procedures. Follow-up images 191 months after the first SRS show stable tumor. The patient's status was stable without neurological deficits.

\section{Discussion}

\section{Observations}

This long-term outcome study describes the management of two patients with invasive, recurrent, and likely fatal meningiomas that progressed within the venous outflow sinuses of the brain. Multimodality management using surgery, radiosurgery, and fractionated radiation therapy led to tumor control in these two patients at intervals of 18 and 21 years after their initial craniotomy.

\section{Lessons}

SRS represents a minimally invasive treatment option designed to stop progression of intracranial meningiomas, either as a primary strategy or as an adjuvant option for recurrent or residual tumors. $^{8-10}$ Multiple reports have since demonstrated 5-year tumor control rates of between $87 \%$ and $98.5 \% .^{11-14} \mathrm{~A}$ comparative study by Mathiesen et al. noted that patients with planned subtotal resection had a tumor recurrence rate of $72 \%$, whereas adjuvant SRS after subtotal resection was associated with a $10 \%$ recurrence rate. ${ }^{15}$ Park et al. observed that SRS as a primary treatment could achieve a tumor control rate of $85 \%$ over 15 years. ${ }^{16}$ Adjuvant radiosurgery helped to significantly reduce the need for more radical surgeries and decrease perioperative complications. ${ }^{12,17,18}$

Kalfas and Scudieri ${ }^{2}$ reported the neurosurgical management of 100 patients with parasagittal and falcine meningiomas who underwent surgery between 1999 and 2013. Twelve patients had venous sinus invasion, and 9 subsequently underwent SRS for residual tumors. Eight of these 9 patients had no tumor progression during a follow-up period that ranged between 2 and 14 years.

In the present case, both patients had complex meningiomas that defied complete resection because of tumor components that invaded dural venous sinuses. During more than a decade of additional care after the initial craniotomy, both patients required repeat radiosurgical procedures and fractionated radiation therapy because of additional tumor progression. Venous sinus involvement in the first patient led to venous congestion of the posterior fossa and subsequent venous vascular compression of the trigeminal nerve. In that patient, neither vascular decompressive surgery nor percutaneous methods to ablate the trigeminal nerve were deemed safe. Instead, SRS was performed to target the root entry zone of the trigeminal nerve. The second case was first published by Deibert and 
Kondziolka $^{19}$ in 2013 to emphasize the complex nature of a recurrent meningioma located in the posterior two-thirds of the sagittal sinus. The case is included in this study because the extended follow-up interval further confirmed the success of long-term tumor control.

The management strategy in these two patients was focused on tumor control and neurological functional preservation despite having only partially resectable tumors at first presentation. Initial surgery, radiosurgery at the time of focal progression, and eventually radiation therapy led to long-term tumor control in these two patients with otherwise incurable tumors occluding venous sinus outflow.

\section{References}

1. Ostrom QT, Gittleman $H$, Liao P, et al. CBTRUS Statistical Report: primary brain and other central nervous system tumors diagnosed in the United States in 2010-2014. Neuro Oncol. 2017;19(suppl 5):v1-v88.

2. Kalfas F, Scudieri $C$. Neurosurgical management of parasagittal and falcine meningiomas: judicious modern optimization of the results in a 100-case study. Asian J Neurosurg. 2019;14(4):1138-1143.

3. Chung SB, Kim CY, Park CK, et al. Falx meningiomas: surgical results and lessons learned from 68 cases. J Korean Neurosurg Soc. 2007;42(4):276-280.

4. Sughrue ME, Rutkowski MJ, Shangari G, et al. Results with judicious modern neurosurgical management of parasagittal and falcine meningiomas. Clinical article. J Neurosurg. 2011;114(3):731-737.

5. Pettersson-Segerlind J, Orrego A, Lönn S, Mathiesen T. Long-term 25-year follow-up of surgically treated parasagittal meningiomas. World Neurosurg. 2011;76(6):564-571.

6. Condra KS, Buatti JM, Mendenhall WM, et al. Benign meningiomas: primary treatment selection affects survival. Int $\mathrm{J}$ Radiat Oncol Biol Phys. 1997;39(2):427-436.

7. van Alkemade $H$, de Leau M, Dieleman EM, et al. Impaired survival and long-term neurological problems in benign meningioma. Neuro Oncol. 2012;14(5):658-666.

8. Kondziolka D, Levy El, Niranjan A, et al. Long-term outcomes after meningioma radiosurgery: physician and patient perspectives. $J$ Neurosurg. 1999;91(1):44-50.

9. Kondziolka D, Mathieu D, Lunsford LD, et al. Radiosurgery as definitive management of intracranial meningiomas. Neurosurgery. 2008;62(1):53-60.

10. Gatterbauer B, Gevsek S, Höftberger R, et al. Multimodal treatment of parasagittal meningiomas: a single-center experience. $J$ Neurosurg. 2017;127(6):1249-1256.
11. Sheehan JP, Lee CC, Xu Z, et al. Edema following Gamma Knife radiosurgery for parasagittal and parafalcine meningiomas. J Neurosurg. 2015;123(5):1287-1293.

12. Lippitz BE, Bartek J Jr, Mathiesen T, Förander P. Ten-year followup after Gamma Knife radiosurgery of meningioma and review of the literature. Acta Neurochir (Wien). 2020;162(9):2183-2196.

13. Kreil W, Luggin J, Fuchs I, et al. Long term experience of Gamma Knife radiosurgery for benign skull base meningiomas. J Neurol Neurosurg Psychiatry. 2005;76(10):1425-1430.

14. Ding D, Xu Z, McNeill IT, et al. Radiosurgery for parasagittal and parafalcine meningiomas. J Neurosurg. 2013;119(4):871-877.

15. Mathiesen T, Pettersson-Segerlind J, Kihlström L, Ulfarsson E. Meningiomas engaging major venous sinuses. World Neurosurg. 2014;81(1):116-124.

16. Park KJ, Kano H, lyer A, et al. Gamma Knife stereotactic radiosurgery for cavernous sinus meningioma: long-term follow-up in 200 patients. J Neurosurg. 2019;130(6):1789-2097.

17. Aboukais R, Zairi F, Reyns N, et al. Surgery followed by radiosurgery: a deliberate valuable strategy in the treatment of intracranial meningioma. Clin Neurol Neurosurg. 2014;124:123-126.

18. Nanda A, Thakur JD, Sonig A, Missios S. Microsurgical resectability, outcomes, and tumor control in meningiomas occupying the cavernous sinus. J Neurosurg. 2016;125(2):378-392.

19. Deibert CP, Kondziolka D. Stereotactic radiosurgery used to manage a meningioma filling the posterior two-thirds of the superior sagittal sinus. J Neurosurg. 2013;119(5):1156-1158.

\section{Disclosures}

L. Dade Lunsford owns stock in Elekta $A B$ and is a consultant for Insightec.

\section{Author Contributions}

Conception and design: Lunsford, Mallela, Faramand. Acquisition of data: Lunsford, Wei, Faramand. Analysis and interpretation of data: Lunsford, Wei, Mallela. Drafting the article: Lunsford, Wei, Mallela, Faramand. Critically revising the article: Lunsford, Wei, Mallela, Niranjan. Reviewed submitted version of manuscript: Lunsford, Wei, Mallela, Niranjan. Approved the final version of the manuscript on behalf of all authors: Lunsford. Administrative/technical/material support: Lunsford, Niranjan. Study supervision: Lunsford.

\section{Correspondence}

L. Dade Lunsford: University of Pittsburgh Medical Center, Pittsburgh, PA. lunsld@upmc.edu. 